\title{
Chemical Ecology and Predator-Prey Interactions: Understanding the Role of Chemistry on Complex, Trophic Relationships in a Changing World
}

\author{
Sara L. Hermann ${ }^{1}$. Jennifer S. Thaler ${ }^{2}$ \\ Received: 25 October 2021 / Revised: 25 October 2021 / Accepted: 28 October 2021 / Published online: 8 November 2021 \\ (c) The Author(s), under exclusive licence to Springer Science+Business Media, LLC, part of Springer Nature 2021
}

A central tenet of ecology lies in disentangling the dynamics between predators and their prey, with a major goal of understanding the mechanisms that mediate their interactions. While predator-prey interactions occur broadly - across taxa, environments, and time scales - one commonality is that chemical ecology plays a sometimes underappreciated role in these interactions. The discovery that chemistry mediates predator-prey interactions, in addition to auditory and visual cues, has allowed us to expand our understanding of prey capture and predator avoidance behaviors. By including chemical information, we can now examine predator-prey relationships over a larger spatial and temporal scale that was previously limited by short-range mechanisms such as sight and sound.

In this special issue, we explore how chemistry interacts with climate change, toxicity, and species interactions and explore the extent to which chemistry plays a role in predator-prey interactions in a food web, multi-trophic context. The selection of articles delves into the two main mechanistic prongs of the antagonistic arms-race between predators and prey: 1) the direct influence of chemistry on individual organisms and 2) the influence of infochemicals in the environment on the interactions between organisms. Importantly, the authors consider the biological context of predator-prey interactions by including crucial considerations of prey host quality, the role of other predators, and importantly, acknowledging the importance of taking a community-level, multi-trophic approach. Further, several of the papers in the collection highlight the growing need to consider abiotic

Sara L. Hermann

Slh@psu.edu

1 Department of Entomology, Program in Ecology, The Pennsylvania State University, University Park, PA, USA

2 Department of Entomology, Cornell University, Ithaca, NY, USA factors arising from global climate change on predator-prey dynamics and the chemistry that influences them.

In recent years, there has been a profusion of research linking global climate change and extreme weather patterns to alterations in species-level interactions (Barton and Ives 2014; Ward et al. 2020; Croy et al. 2021). It is clear that the impact of climate change is multifaceted and thus we expect it to affect predator-prey interactions by changing species both directly and by changing their interactions with conspecifics and heterospecific organisms (Barton 2010; Miller et al. 2017; Kansman et al. 2021). It is important to predict abiotic stress that organisms will face in the future and test the implications of that stress. For example, Guyer et al. 2021 used climate models to experimentally test how climate change will affect interactions between maize, its herbivorous pest (Diabrotica balteata), and entomopathogenic nematode predators. They found dynamic effects of changing plant quality on both the herbivores and the predators and predict that overall biological control will be weaker in the future climate. In addition to climate, altered $\mathrm{CO}_{2}$ can also influence organisms both directly and indirectly. Increasing $\mathrm{CO}_{2}$ in the atmosphere reduces $\mathrm{pH}$ and thus is linked to ocean acidification. While acidification itself can have important implications for individual organisms (Talmage and Gobler 2010; Heuer and Grosell 2014; Schirrmacher et al. 2021), found that olfactory perception is $\mathrm{pH}$ dependent and thus, decreasing $\mathrm{pH}$ with increasing atmospheric $\mathrm{Co} 2$ affects the detection and behavioral orientation toward predator-produced chemicals. These studies highlight the importance of considering not only the direct impact of climate change on species interactions but also how physical and chemical mechanisms that underlie interactions will be affected by abiotic fluctuations.

Articles in this special issue also advance our understanding of the mechanisms of toxicity in predator-prey interactions, in terms of predator resistance to prey toxicity, variation in sequestration of toxins from prey organisms, 
and how to appropriately measure toxicity in predator-prey systems. Resistance to predator venom can alter population dynamics by allowing for prey to escape from predation. However, the mechanisms for venom resistance are not wellunderstood. To address this, Rodrigues et al. 2021 measured venom resistance in Boa constrictors which are predators that can themselves be eaten by venomous coral snakes. Their results demonstrate that boas have evolved resistance to venom using phospholipase inhibitors in their blood that reduce several mechanisms of venom toxicity, providing valuable information for modeling species interactions and population dynamics of these snakes. Additionally, some predator species use toxic prey to their advantage through sequestration-a strategy that allows predators to be chemically defended by higher trophic levels. Yet, research assessing sequestration in wild animals over the species range is limited. Mori et al. collected the Japanese natracine snake that sequesters bufodienolides from its toad prey across Japan and found geographic variation in their sequestration, highlighting the importance of measuring toxicity outside of controlled lab settings. In a laboratory study, Vilar et al. 2021 investigated the presumably defensive toxins that are induced in cyanobacterial prey when exposed to infochemicals from grazing Daphnia. Here, Daphnia cues induced cyanotoxins in the prey, but surprisingly, this production of toxins had no detectable fitness cost for the cyanobacteria. The improvement of analytical techniques and experimental methodology is the backbone for advances in chemical ecology. Chan et al. 2021 specifically addressed the appropriateness of using a model system (Brine shrimp), over ecologically relevant predators to assess prey toxicity. The authors found that the use of this model system proved to be an acceptable and reliable option that reduces limitations and allows for quick results compared to using vertebrate predators. Developing model systems for ecological experimentation is valuable for identifying mechanisms driving interactions. However, there is a need for ecologically relevant, field-based studies to validate lab-based results, especially as it pertains to toxicity in predator-prey interactions-as this was a common theme across the articles in the issue.

Another critical advancement in chemical ecology of predator-prey interactions is the discussion of how hostplant chemistry affects third trophic level consumers, and how predator chemical information is recognized by other species within the environment. To illustrate the influence of chemistry in a tri-trophic system, Ugine et al. 2021 assessed how prey quality varies based on the species and nutritional content of the host plant. The authors demonstrated poor quality prey, as a function of host plant quality, contributed to a shift to omnivory in the third trophic level. To further understand the impact of an infochemical on predators and prey, Grunseich et al. 2021 measured cues released by entomopathogenic nematodes and examined how these cues are used by competing predators and their cucumber beetle prey. Foraging prey were able to differentiate between the cues of the predators and responded by avoiding the actively hunting nematodes. Interestingly, competing predators used the heterospecific chemical information to locate areas with potential prey. This work highlights the importance of investigating predator-prey chemical ecology through a multitrophic lens to better understand the species-level variation in detection, response, and interactive effects of infochemicals on food webs.

It is a time of rapid discovery in the field of predator-prey ecology as we continue to identify chemical mechanisms that mediate interactions and delve into the complexity of the systems, beyond dyadic relationships, to better understand potential cascading implications. This Special Issue highlights advances in a wide array of taxa from plants and insects to plankton and snakes, and environments ranging from marine to terrestrial. Indeed, great progress is being made but future work must continue to focus on understanding how a changing climate is interacting with and modifying species interactions. In addition, it is clear that bringing research to the field, in realistic settings, will allow us to observe important variation in responses, and consider the community-wide implications of predator-prey interactions that are modulated by chemical ecology.

\section{Declarations}

Competing Interests The authors declare no competing interests.

\section{References:}

BT Barton 2010 Climate warming and predation risk during herbivore ontogeny Ecology 9128112818

BT Barton AR Ives 2014 Species interactions and a chain of indirect effects driven by reduced precipitation Ecology 95486494

Chan W, Shaughnessy AEP, van den Berg CP, et al (2021) The Validity of Brine Shrimp (Artemia Sp.) Toxicity Assays to Assess the Ecological Function of Marine Natural Products. J Chem Ecol. https://doi.org/10.1007/s10886-021-01264-Z

JR Croy JD Pratt D Sheng KA Mooney 2021 Climatic displacement exacerbates the negative impact of drought on plant performance and associated arthropod abundance Ecology $102 \mathrm{e} 03462$

Grunseich JM, Aguirre NM, Thompson MN, et al (2021) Chemical Cues from Entomopathogenic Nematodes Vary Across Three Species with Different Foraging Strategies, Triggering Different Behavioral Responses in Prey and Competitors. J Chem Ecol. https://doi.org/10.1007/s10886-021-01304-8

Guyer A, van Doan C, Maurer C, et al (2021) Climate Change Modulates Multitrophic Interactions Between Maize, A Root Herbivore, and Its Enemies. Journal of Chemical Ecology

RM Heuer M Grosell 2014 Physiological impacts of elevated carbon dioxide and ocean acidification on fish Am J Physiol Regul Integr Comp Physiol 307 R1061 R1084 
T Inoue R Nakata AH Savitzky 2021 New Insights Into Dietary Toxin Metabolism: Diversity in the Ability of the Natricine Snake Rhabdophis tigrinus to Convert Toad-Derived Bufadienolides J Chem Ecol https://doi.org/10.1007/s10886-021-01287-6

JT Kansman DW Crowder DL Finke 2021 Primacy of plants in driving the response of arthropod communities to drought Oecologia 195833842

CR Miller BT Barton L Zhu 2017 Combined effects of night warming and light pollution on predator-prey interactions Proc Biol Sci 284 20171195 https://doi.org/10.1098/rspb.2017.1195

Rodrigues CFB, Zdenek CN, Serino-Silva C, et al (2021) BoayPLI from Boa constrictor Blood is a Broad-Spectrum Inhibitor of Venom PLA2 Pathophysiological Actions. Journal of Chemical Ecology

P Schirrmacher CC Roggatz DM Benoit JD Hardege 2021 Ocean Acidification Amplifies the Olfactory Response to 2-Phenylethylamine: Altered Cue Reception as a Mechanistic Pathway? J Chem Ecol https://doi.org/10.1007/s10886-021-01276-9
SC Talmage CJ Gobler 2010 Effects of past, present, and future ocean carbon dioxide concentrations on the growth and survival of larval shellfish Proc Natl Acad Sci U S A 1071724617251

TA Ugine HK Gill N Hernandez 2021 Predator Performance and Fitness Is Dictated by Herbivore Prey Type Plus Indirect Effects of their Host Plant J Chem Ecol https://doi.org/10.1007/ s10886-021-01251-4

Vilar MCP, da Costa Pena Rodrigues TF, da Silva Ferrão-Filho A, de Oliveira e Azevedo SMF (2021) Grazer-Induced Chemical Defense in a Microcystin-Producing Microcystis aeruginosa (Cyanobacteria) Exposed to Daphnia gessneri Infochemicals. Journal of Chemical Ecology

SF Ward BH Aukema S Fei AM Liebhold 2020 Warm temperatures increase population growth of a nonnative defoliator and inhibit demographic responses by parasitoids Ecology 101 e03156 